\title{
Blood pressure components and risk for chronic kidney disease in middle-aged Japanese men: The Kansai Healthcare Study
}

\author{
Hideo Koh ${ }^{1}$, Tomoshige Hayashi ${ }^{1}$, Kyoko Kogawa Sato ${ }^{1}$, Nobuko Harita ${ }^{1}$, Isseki Maeda ${ }^{1}$, Yoshiko Nakamura ${ }^{2}$, \\ Ginji Endo ${ }^{1}$, Hiroshi Kambe ${ }^{2}$ and Kanji Fukuda ${ }^{1,2}$
}

It is unclear which blood pressure (BP) components (that is, systolic BP (SBP), diastolic BP (DBP), pulse pressure (PP) and mean arterial pressure (MAP)) are superior predictors of chronic kidney disease (CKD). Furthermore it is unclear whether the combination of SBP+DBP or PP+MAP is superior to any of these four individual BP components in predicting CKD. We enrolled 9928 Japanese men aged 40-55 years who had a normal estimated glomerular filtration rate (eGFR), no proteinuria and no history of cardiovascular disease and were not taking any antihypertensive medications at baseline. CKD was defined as an eGFR of $<60 \mathrm{ml} \mathrm{min}^{-1}$ per $1.73 \mathrm{~m}^{2}$ using the modified diet in renal disease equation. $\Delta$ Akaike's information criterion ( $\left.\triangle A I C\right)$ was used to compare the BP components-added model to the model without them in a Cox proportional hazards model. During the 52428 person-years of follow-up, there were 434 cases of CKD. Of all four BP components, the model including DBP- or MAP-alone had the highest values of $\triangle A I C$ (10.2 and 9.85, respectively). The PP-alone model had the lowest $\triangle A I C$ value $(-1.48)$. The combination models including SBP+DBP ( $\triangle \mathrm{AIC}$ 8.42) or PP+MAP (8.42) were not superior to the models including DBP-or MAP-alone. These findings suggested that, of the four BP components, both DBP and MAP were the most useful predictors for subsequent incidence of CKD, but PP was not an important predictor. The combination model, including SBP+DBP or PP+MAP, was not superior to the models including DBP- or MAP-alone for predicting CKD.

Hypertension Research (2011) 34, 536-541; doi:10.1038/hr.2011.2; published online 27 January 2011

Keywords: blood pressure component; chronic kidney disease; estimated glomerular filtration rate; middle-aged men; prospective cohort study

\section{INTRODUCTION}

Hypertension is a well-known risk factor for cardiovascular diseases, ${ }^{1,2}$ and it has also been associated with the incidence of chronic kidney disease (CKD).$^{3-7}$ Some epidemiological studies have focused on which blood pressure (BP) components are superior as predictors of cardiovascular diseases, including systolic BP (SBP), diastolic BP (DBP), pulse pressure (PP) and mean arterial pressure (MAP).$^{8-17}$ However, the BP components that are the most important risks factor for CKD are currently unknown.

A number of epidemiological studies have examined the association between hypertension, defined by SBP and DBP, and the risk of $\mathrm{CKD}^{3-7} \mathrm{BP}$ is also divided into two other components: MAP, which reflects a steady component, and $\mathrm{PP}$, which reflects a pulsatile component. ${ }^{18-21} \mathrm{PP}$ is mainly determined by ventricular ejection, arterial stiffness and the timing of the reflected waves, and it increases with advancing age. ${ }^{18-21}$ MAP mainly depends on cardiac output and peripheral vascular resistance. ${ }^{18-21}$ Only two prospective studies have examined associations between these four BP components and the incidence of reduced kidney function. ${ }^{22,23}$ One study focused on only subjects over the age of 65 with isolated systolic hypertension and did not include subjects with diastolic hypertension or normotension. ${ }^{22}$ The other study used data based on self-reported BP information. ${ }^{23}$ No prospective cohort study using both normotensive and hypertensive subjects has examined the relationship of directly measured BP components, such as SBP, DBP, PP and MAP, to the incidence of CKD (defined by low estimated glomerular filtration rate (eGFR)).

We therefore examined the effects of SBP, DBP, PP and MAP on the onset of CKD, as defined by an eGFR of $<60 \mathrm{ml} \mathrm{min}^{-1}$ per $1.73 \mathrm{~m}^{2}$ in middle-aged Japanese men. Our specific purposes were: (1) to examine which BP components (that is, SBP, DBP, PP and MAP) were superior predictors of $\mathrm{CKD}$ and (2) to examine whether it was more helpful to use combined $\mathrm{BP}$ components (that is, SBP+DBP or PP+MAP) to predict the risk of CKD instead of any one of the four single BP components.

${ }^{1}$ Department of Preventive Medicine and Environmental Health, Osaka City University Graduate School of Medicine, Osaka, Japan and ${ }^{2}$ Kansai Health Administration Center, Nippon Telegraph and Telephone West Corporation, Osaka, Japan

Correspondence: Dr T Hayashi, Department of Preventive Medicine and Environmental Health, Osaka City University Graduate School of Medicine, 1-4-3, Asahi-machi, Abeno-ku, Osaka 545-8585, Japan.

E-mail: thayashi@med.osaka-cu.ac.jp

Received 9 June 2010; revised 14 October 2010; accepted 10 November 2010; published online 27 January 2011 


\section{METHODS}

\section{Study sample}

The Kansai Healthcare Study was an ongoing cohort investigation designed to examine risk factors for cardiometabolic diseases. The details of the study have been described previously. ${ }^{24-26}$ Between April 2000 and March 2001, 12647 male employees of a company in Kansai, Japan, aged 40-55 years at entry and considered to be involved in sedentary jobs were enrolled in this study. All employees in this company aged 40 years or older undergo annual medical checkups. The protocol for this research was reviewed by the Human Subjects Review Committee at Osaka City University.

Eligibility criteria for inclusion in the study were as follows: an eGFR of $60 \mathrm{ml} \mathrm{min}^{-1}$ per $1.73 \mathrm{~m}^{2}$ or higher; dipstick-negative proteinuria; no history of cardiovascular diseases; and not taking oral antihypertensive medications at baseline. A total of 10666 Japanese men were included in this study. Of them, we excluded 349 men who had missing covariate information at baseline. We also excluded 389 participants because they did not undergo annual checkups during the follow-up period. Thus, the analytic cohort consisted of 9928 men.

\section{Data collection and measurements}

The clinical examination consisted of the following: medical history; physical examination; anthropometric measurements; self-reported questionnaires on lifestyle characteristics, such as regular leisure-time physical activity, smoking habit and daily alcohol consumption; measurements of fasting plasma glucose and serum creatinine; and dipstick urinalysis. Trained nurses took all of the measurements. Blood samples were drawn after an overnight 12-h fast, and plasma glucose was measured. Diabetes was diagnosed if the fasting plasma glucose level was $\geqslant 126 \mathrm{mg} \mathrm{dl}^{-1}$ or if participants were taking oral hypoglycemic medications or insulin according to the American Diabetes Association criteria. ${ }^{27}$ Serum creatinine was measured enzymatically using a Hitachi 7350 automatic chemistry analyzer (Hitachi, Tokyo, Japan). Serum creatinine at baseline was also measured by the Jaffe method in 1930 subjects. We recalibrated the values of the Jaffe method to the values of the enzymatic method using the following formula: serum creatinine $\left(\mathrm{mg} \mathrm{dl}^{-1}\right.$, enzymatic method $)=1.02 \times$ serum creatinine $\left(\mathrm{mg} \mathrm{dl}^{-1}\right.$, Jaffe method) 0.25 ( $\left.r=0.9996\right)$. We calculated eGFR using the Modification of Diet in Renal Disease (MDRD) Study equation for Japanese men, ${ }^{28}$ which was validated by the standard inulin clearance techniques, as follows: $\mathrm{GGFR}=194 \times \mathrm{age}^{-0.287} \times$ serum creatinine $^{-1.094}$. The serum creatinine in the equation was the value measured by the enzymatic method. CKD was defined as an eGFR $<60 \mathrm{ml} \mathrm{min}^{-1}$ per $1.73 \mathrm{~m}^{2}$. CKD was diagnosed based on the eGFR level at baseline and each annual follow-up examination. Dipstick urinalysis was occasionally performed on fresh and midstream urine. The results of the dipstick urinalysis were interpreted as negative, , $\pm 1+, 2+, 3+$ or $4+$. Proteinuria was defined as $1+$ or higher at baseline. Body mass index was calculated as weight in $\mathrm{kg}$ divided by height in $\mathrm{m}^{2}$.

After a 5-min rest period in a quiet room, SBP and DBP were measured in a sitting position on the right arm with an automatic sphygmomanometer (BP-203RV; Omron Colin, Tokyo, Japan, and Udex-super; ELK Osaka, Japan). The Spearman's correlation between the former device and a standard mercury sphygmomanometer was 0.985 for SBP and 0.976 for DBP. The Spearman's correlation between the latter device and a standard mercury sphygmomanometer was 0.997 for SBP and 0.976 for DBP. BP was measured once or twice at an interval of a few minutes. If the initial reading showed hypertension, BP was measured again, and the subject's BP was defined as the lower of the two readings after the second measurement. Hypertension was defined as SBP $\geqslant 140 \mathrm{mmHg}$ or DBP $\geqslant 90 \mathrm{~mm} \mathrm{Hg.}{ }^{1}$ PP was defined as SBP minus DBP. MAP was calculated as DBP plus one-third PP.

Questions about alcohol intake included the weekly frequency of alcohol consumption and the usual amount of alcohol consumed on a daily basis. The types of beverage included beer, sake (rice wine), whiskey and shochu (a type of brandy). The units of alcohol consumed were expressed as ' $g o$ ', or the equivalent of $23.0 \mathrm{~g}$ of ethanol, and were converted to daily alcohol consumption (in $\mathrm{g}$ of ethanol per day). One go is $180 \mathrm{ml}$ of sake, which corresponds to one bottle $(663 \mathrm{ml})$ of beer, two single shots $(75 \mathrm{ml})$ of whisky, or a half $g o$ $(90 \mathrm{ml})$ of shochu. Regarding smoking habits, participants were classified as non-smokers, past smokers or current smokers. The single-item questionnaire regarding leisure-time physical activity had three possible answers: rarely, sometimes and regular (that is, at least once weekly). Participants were classified as engaging in regular leisure-time physical activity at least once weekly or less than once weekly. The validity of this questionnaire has been described in detail previously. ${ }^{24}$

\section{Statistical analysis}

We used unpaired $t$-tests for continuous variables or $\chi^{2}$-tests for categorical variables to examine the difference between participants who developed CKD and those who did not. We used Cox proportional hazards models to investigate the association between $\mathrm{BP}$ components and the incidence of CKD. Follow-up of each subject was continued until diagnosis of CKD or until 31 March 2007, which ever came first. The proportional hazards assumption was confirmed by a log-minus-log plot. Nonlinear effects of continuous independent variables were evaluated by categorizing a continuous variable into multiple dichotomous variables of equal units and visually assessing a scatter plot of each variable's coefficient in the Cox proportional hazards models against the median value of each class of dichotomous variables. ${ }^{29}$ The presence of an effect modification was tested by the insertion of first-order interaction terms into appropriate regression models. We calculated the $95 \%$ confidence interval for each hazard ratio.

In the Cox proportional hazards models, $\Delta$ Akaike's information criterion ( $\Delta$ AIC) and the $\Delta \chi^{2}$ of likelihood ratio test were used as a measure of the improvement of goodness of fit to compare the two models, one of which was nested within the other. We used $\triangle \mathrm{AIC}$ to evaluate which BP components were superior predictors of the risk for the incidence of CKD and whether the models including combined $\mathrm{BP}$ components (that is, $\mathrm{SBP}+\mathrm{DBP}$ or $\mathrm{PP}+\mathrm{MAP}$ ) outperformed models including any one of the four single BP components for predicting the incidence of CKD because AIC can be used to compare non-nested models. An absolute $\triangle \mathrm{AIC}$ value of $\leqslant 2$ indicates no meaningful difference of goodness of fit between the methods. An absolute $\triangle$ AIC value of $>2$ indicates a meaningful difference of goodness of fit between the methods. ${ }^{30}$ A larger decrease in AIC can be interpreted as a better fit. In models including combined $\mathrm{BP}$ components (SBP+DBP or PP+MAP), we assessed multicollinearity by inspecting the changes in the coefficient and standard error of each BP component variable caused by the addition of the other. $^{31}$ The linear trends in risks of quartiles of all BP components were evaluated by entering indicators for each categorical level of exposure using the median value for each category.

All $P$ values were two-tailed and considered statistically significant if the values were $<0.05$. All statistical analyses were performed using PASW Statistics, version 17.0 (SPSS, Chicago, IL, USA).

\section{RESULTS}

The baseline characteristics of the study participants are shown in Table 1. During the 52428 person-years of follow-up (a median follow-up of 6.0 years), there were 434 incident cases of CKD. Participants who developed CKD had higher mean values of SBP, DBP and MAP than those who did not (Table 1). There was no significant difference in PP between the groups. The group of participants who developed CKD also had a higher proportion of hypertension, a higher mean body mass index and a lower mean eGFR at baseline (Table 1).

In the Cox proportional hazards models, all continuous variables, except eGFR, in the models (presented in Tables 2 and 3) fulfilled the linearity assumption. To account for the nonlinearity of eGFR, we fit a model using categorized eGFR (60.0-69.9, 70.0-79.9, 80.0-89.9, $\geqslant 90.0 \mathrm{ml} \mathrm{min}^{-1}$ per $1.73 \mathrm{~m}^{2}$ ) in all models presented in Tables 2 and 3. Therefore, the following variables were included in the base model: age, body mass index, diabetes status (non-diabetes or diabetes), daily alcohol consumption, regular leisure-time physical activity (yes/no), smoking habit (non-smokers, past smokers, or current smokers), and categorized eGFR (60.0-69.9, 70.0-79.9, $80.0-89.9$, $\geqslant 90.0 \mathrm{ml} \mathrm{min}^{-1}$ per $1.73 \mathrm{~m}^{2}$ ). We examined the significance of the interaction terms $\mathrm{SBP} \times \mathrm{DBP}$ and $\mathrm{PP} \times \mathrm{MAP}$ in all models 
Table 1 Baseline characteristics of study participants according to whether CKD developed during the follow-up period

\begin{tabular}{|c|c|c|c|}
\hline Characteristics & $C K D(-)$ & $C K D(+)$ & $\mathrm{P}$ value \\
\hline$n$ & 9494 & 434 & \\
\hline Age, years & $47.8 \pm 4.2$ & $48.6 \pm 4.1$ & $<0.001$ \\
\hline Body mass index, $\mathrm{kg} \mathrm{m}^{-2}$ & $23.3 \pm 2.9$ & $23.9 \pm 2.9$ & $<0.001$ \\
\hline Systolic blood pressure, $\mathrm{mm} \mathrm{Hg}$ & $127.9 \pm 17.9$ & $130.9 \pm 18.3$ & 0.001 \\
\hline Diastolic blood pressure, $\mathrm{mm} \mathrm{Hg}$ & $79.7 \pm 11.9$ & $82.5 \pm 11.5$ & $<0.001$ \\
\hline Pulse pressure, mm Hg & $48.1 \pm 12.6$ & $48.5 \pm 13.1$ & 0.626 \\
\hline Mean arterial pressure, $\mathrm{mm} \mathrm{Hg}$ & $95.8 \pm 12.9$ & $98.6 \pm 12.7$ & $<0.001$ \\
\hline Hypertension, \% & $2722(28.7)$ & $158(36.4)$ & 0.001 \\
\hline Fasting plasma glucose, $\mathrm{mg} \mathrm{dl}^{-1}$ & $101.3 \pm 21.0$ & $99.2 \pm 17.1$ & 0.036 \\
\hline Diabetes, \% & $653(6.9)$ & $19(4.4)$ & 0.043 \\
\hline Alcohol consumption, g ethanol per day & $25.6 \pm 22.0$ & $21.3 \pm 20.0$ & $<0.001$ \\
\hline Drinking habits, \% & $8105(85.4)$ & $357(82.3)$ & 0.074 \\
\hline Regular leisure-time physical activity, \% & $1704(17.9)$ & $91(21.0)$ & 0.110 \\
\hline Current smoker, \% & $5600(59.0)$ & 199 (45.9) & $<0.001$ \\
\hline Serum creatinine, $\mathrm{mg} \mathrm{dl}^{-1}$ & $0.82 \pm 0.13$ & $0.98 \pm 0.12$ & $<0.001$ \\
\hline eGFR, $\mathrm{ml} \mathrm{min}^{-1}$ per $1.73 \mathrm{~m}^{2}$ & $86.2 \pm 14.2$ & $69.7 \pm 9.8$ & $<0.001$ \\
\hline
\end{tabular}

Abbreviations: CKD, chronic kidney disease; eGFR, estimated glomerular filtration rate. Data are mean $\pm s . d$. or $n(\%)$

Table 2 Comparison of four blood pressure components and their combinations in predicting the incidence of chronic kidney disease

\begin{tabular}{|c|c|c|c|c|}
\hline \multirow[b]{2}{*}{ Model 1} & \multirow[b]{2}{*}{ Model 2} & \multirow[b]{2}{*}{$\triangle A I C$} & \multicolumn{2}{|c|}{ Likelihood ratio } \\
\hline & & & $\Delta \chi^{2}$ & $\mathrm{P}$ value \\
\hline \multicolumn{5}{|c|}{ The effects of adding each BP component to base model } \\
\hline Base $^{a}$ & Base $^{\mathrm{a}}+\mathrm{SBP}$ & 5.56 & 7.56 & 0.006 \\
\hline Base $^{a}$ & Base $^{\mathrm{a}}+\mathrm{DBP}$ & 10.2 & 12.19 & $<0.001$ \\
\hline Base $^{a}$ & Base $^{a}+P P$ & -1.48 & 0.52 & 0.471 \\
\hline Base $^{a}$ & Base $^{\mathrm{a}}+\mathrm{MAP}$ & 9.85 & 11.85 & 0.001 \\
\hline Base $^{a}$ & Base $^{\mathrm{a}}+\mathrm{SBP}+\mathrm{DBP}$ & 8.42 & 12.43 & 0.002 \\
\hline Base $^{a}$ & Base $^{a}+P P+M A P$ & 8.42 & 12.43 & 0.002 \\
\hline \multicolumn{5}{|c|}{ The effects of adding the second BP component to each model } \\
\hline Base $^{\mathrm{a}}+\mathrm{SBP}$ & Base $^{\mathrm{a}}+\mathrm{SBP}+\mathrm{DBP}$ & 2.87 & 4.87 & 0.027 \\
\hline Base $^{a}+$ DBP & Base $^{\mathrm{a}}+\mathrm{DBP}+\mathrm{SBP}$ & -1.76 & 0.24 & 0.625 \\
\hline Base $^{a}+P P$ & Base $^{a}+P P+M A P$ & 9.91 & 11.91 & 0.001 \\
\hline Base $^{\mathrm{a}}+\mathrm{MAP}$ & Base $^{a}+M A P+P P$ & -1.42 & 0.58 & 0.447 \\
\hline
\end{tabular}

Abbreviations: AIC indicates Akaike's information criterion; CKD, chronic kidney disease; $\mathrm{DBP}$, diastolic blood pressure; MAP, mean arterial pressure; PP, pulse pressure;

SBP, systolic blood pressure.

Likelihood ratio $\Delta \chi^{2}$ statistics and $\Delta \mathrm{AIC}$ for chronic kidney disease outcomes were calculated by comparing model 2 to model 1 . $\triangle A I C$ was used to evaluate which blood pressure components or combined BP components, SBP, DBP, PP, MAP, SBP+DBP or PP+MAP, were superior as the risk for incident CKD. Higher value of $\triangle A I C$ indicates better model fit and predictive utility. Likelihood ratio $\Delta \chi^{2}$ statistics and its $P$ values were used as a measure of the improvement of goodness of fit to compare the fit between model 1 and model 2, one of which was nested within the other.

aBase model includes age, body mass index, diabetes status (non-diabetes or diabetes), daily alcohol consumption, regular leisure-time physical activity (yes/no), smoking habits (nonsmokers, past smokers or current smokers) and estimated glomerular filtration rate categories $\left(60.0-69.9,70.0-79.9,80.0-89.9, \geqslant 90.0 \mathrm{ml} \mathrm{min}^{-1}\right.$ per $\left.1.73 \mathrm{~m}^{2}\right)$ at baseline, but no BP component.

including these two BP components (Tables 2 and 3). None of these interactions were significant. In all models presented in Tables 2 and 3 , the proportional hazards assumption was confirmed by a log-minuslog plot, and all independent variables met the assumption.

To examine which BP components or combined BP components (that is, SBP, DBP, PP, MAP, SBP+DBP or PP+MAP) were superior predictors of incident $\mathrm{CKD}, \triangle \mathrm{AIC}$ was calculated as the AIC of the
Table 3 Comparison of four blood pressure components and their combinations in predicting the incidence of chronic kidney disease

\begin{tabular}{lcr}
\hline \multicolumn{4}{c}{$\begin{array}{c}\text { Hazard ratio } \\
(95 \% \mathrm{Cl})\end{array}$} & P value \\
\hline Mode ${ }^{\circ}$ & & \\
\hline Continuous analysis & & \\
Multiple-adjusted models including a single BP component & \\
SBP, per 1 s.d. & $1.14(1.04-1.26)$ & 0.005 \\
DBP, per 1 s.d. & $1.20(1.08-1.32)$ & $<0.001$ \\
PP, per 1 s.d. & $1.04(0.94-1.14)$ & 0.469 \\
MAP, per 1 s.d. & $1.19(1.08-1.31)$ & $<0.001$ \\
Multiple-adjusted models including combined two BP components & \\
SBP and DBP & & \\
SBP, per 1 s.d. & $1.03(0.91-1.18)$ & 0.624 \\
DBP, per 1 s.d. & $1.17(1.02-1.34)$ & 0.028 \\
PP and MAP & & \\
PP, per 1 s.d. & $0.96(0.87-1.06)$ & 0.448 \\
MAP, per 1 s.d. & $1.21(1.09-1.35)$ & 0.001
\end{tabular}

Categorical analysis

Multiple-adjusted models including a single BP component $\mathrm{SBP}, \mathrm{mm} \mathrm{Hg}$

Quartile $1(-116)$

1.00 (reference)

Quartile 2 (117-126)

$1.23(0.92-1.65)$

Quartile 3 (127-138)

$1.41(1.07-1.88)$

Quartile 4 (139-)

$1.51(1.13-2.02)$

0.017

$P$ for trend

0.004

DBP, $\mathrm{mm} \mathrm{Hg}$

Quartile $1(-71)$

1.00 (reference)

Quartile 2 (72-80)

$1.25(0.92-1.68)$

$1.45(1.08-1.96)$

0.149

Quartile 3 (81-87)

$1.75(1.30-2.37)$

0.015

Quartile 4 (88-)

$<0.001$

$\mathrm{PP}, \mathrm{mm} \mathrm{Hg}$

Quartile $1(-40)$

Quartile 2 (41-47)

1.00 (reference)

Quartile 3 (48-55)

$0.84(0.64-1.10)$

$0.93(0.71-1.21)$

Quartile 4 (56-)

$1.05(0.81-1.35)$

0.734

$P$ for trend

0.606

MAP, $\mathrm{mm} \mathrm{Hg}$

Quartile $1(-86.7)$

1.00 (reference)

Quartile $2(86.8-95.2)$

$1.20(0.89-1.63)$

0.231

Quartile 3 (95.3-103.7)

$1.37(1.02-1.83)$

0.038

Quartile 4 (103.8-)

$1.65(1.23-2.22)$

0.001

$P$ for trend $<0.001$

Multiple-adjusted models including combined two BP components SBP and DBP

$\mathrm{SBP}, \mathrm{mm} \mathrm{Hg}$

Quartile $1(-116)$

Quartile 2 (117-126)

1.00 (reference)

Quartile 3 (127-138)

$1.12(0.82-1.52)$

Quartile 4 (139-)

$P$ for trend

$1.18(0.86-1.63)$

$1.15(0.81-1.64)$

0.493

DBP, $\mathrm{mm} \mathrm{Hg}$

Quartile $1(-71)$

Quartile 2 (72-80)

1.00 (reference)

$1.20(0.87-1.64)$

0.266

Quartile 3 (81-87)

$1.35(0.96-1.88)$

0.081

Quartile 4 (88-)

$1.60(1.11-2.32)$

0.012

$P$ for trend

0.009

$\mathrm{PP}$ and MAP

$\mathrm{PP}, \mathrm{mm} \mathrm{Hg}$

Quartile $1(-40)$

Quartile 2 (41-47)

1.00 (reference)

$0.80(0.61-1.05)$ 
Table 3 (Continued)

\begin{tabular}{|c|c|c|}
\hline Modep & $\begin{array}{c}\text { Hazard ratio } \\
(95 \% \mathrm{Cl})\end{array}$ & $\mathrm{P}$ value \\
\hline Quartile 3 (48-55) & $0.87(0.66-1.13)$ & 0.292 \\
\hline Quartile 4 (56-) & $0.90(0.69-1.18)$ & 0.447 \\
\hline$P$ for trend & 0.560 & \\
\hline \multicolumn{3}{|l|}{ MAP, mm Hg } \\
\hline Quartile 1 (-86.7) & 1.00 (reference) & \\
\hline Quartile 2 (86.8-95.2) & $1.22(0.90-1.66)$ & 0.193 \\
\hline Quartile 3 (95.3-103.7) & $1.40(1.04-1.89)$ & 0.027 \\
\hline Quartile 4 (103.8-) & $1.70(1.24-2.31)$ & 0.001 \\
\hline$P$ for trend & $<0.001$ & \\
\hline
\end{tabular}

Abbreviations: DBP, diastolic blood pressure; MAP, mean arterial pressure; PP, pulse pressure; SBP, systolic blood pressure; s.d., standard deviation; $95 \% \mathrm{Cl}, 95 \%$ confidence interval. ${ }^{a}$ Adjusted for age, body mass index, diabetes status (non-diabetes or diabetes), daily alcohol consumption, regular leisure-time physical activity (yes/no), smoking habits (non-smokers, pas smokers or current smokers) and estimated glomerular filtration rate categories (60.0-69.9 $70.0-79.9,80.0-89.9, \geqslant 90.0 \mathrm{ml} \mathrm{min}^{-1}$ per $1.73 \mathrm{~m}^{2}$ ) at baseline.

model that did not include any BP components in model 1 (Table 2) minus the AIC of the model that included any one BP component or combined BP components in model 2 (Table 2). Of all of the models including each BP component, those including DBP- or MAP-alone had the strongest utility for the prediction of incident CKD ( $\triangle \mathrm{AIC}$ : 10.2 and 9.85, respectively). By contrast, PP had no utility for predicting the incidence of $\mathrm{CKD}(\triangle \mathrm{AIC}=-1.48)$. Furthermore, the model containing both SBP and DBP $(\triangle \mathrm{AIC}=8.42)$ was not superior to the DBP-alone model $(\triangle \mathrm{AIC}=10.2)$ in predicting the incidence of $\mathrm{CKD}$. The model containing both MAP and PP $(\triangle \mathrm{AIC}=8.42)$ was also not superior to the MAP-alone model $(\triangle \mathrm{AIC}=9.85)$ in predicting the incidence of CKD.

The $\Delta \mathrm{AIC}$ and the $\Delta \chi^{2}$ of the likelihood ratio test were used as measures of the improvement of goodness of fit to compare the two models, one of which was nested within the other (Table 2). When SBP was added to the DBP model, the model fit was not improved $(\triangle \mathrm{AIC}=-1.76$, likelihood ratio test, $P=0.625)$. However, when DBP was added to the SBP model, the model fit was improved $(\triangle \mathrm{AIC}=2.87$, likelihood ratio test, $P=0.027)$. Therefore, combining SBP with DBP provided no additional information to the DBP-alone model for predicting the incidence of CKD. When PP was added to the MAP model, the model fit was not improved $(\triangle \mathrm{AIC}=-1.42$, likelihood ratio test, $P=0.447)$, but when MAP was added to the PP model, the model fit was improved $(\triangle \mathrm{AIC}=9.91$, likelihood ratio test, $P=0.001)$. Combining PP and MAP provided no additional information to the MAP-alone model for predicting the incidence of CKD.

The multiple-adjusted hazard ratios of each BP component for the incidence of CKD are shown in Table 3 and presented by 1 s.d. increase and by the quartile of each BP component. DBP and MAP had the highest hazard ratios by 1 s.d. increase for the incidence of CKD. When SBP and DBP were included simultaneously in the model, DBP, but not SBP, was associated with an increased risk of CKD. When $\mathrm{PP}$ and MAP were included simultaneously in the model, MAP, but not PP, was associated with an increased risk of CKD.

\section{DISCUSSION}

These prospective data demonstrate that of all of the four BP components, DBP and MAP were the most useful predictors for risk of subsequent incidence of CKD. Neither combination model (SBP+DBP or PP+MAP) was superior to either the DBP-alone or the MAP-alone model for predicting the incidence of CKD. Models including SBP+DBP or PP+MAP had similar predictive values for the incidence of CKD. These findings were independent of age, body mass index, diabetes status, daily alcohol consumption, regular leisure-time physical activity, smoking habit and baseline eGFR.

A limited number of prospective studies have investigated the associations between the four BP components SBP, DBP, PP, and MAP and the risk of reduced kidney function. ${ }^{22,23}$ Young et al. ${ }^{22}$ showed in the Systolic Hypertension in the Elderly Program that SBP was associated with the highest risk of decline of kidney function as defined by an increase in serum creatinine to $\geqslant 0.4 \mathrm{mg} \mathrm{dl}^{-1}$ at follow-up compared with the baseline level. As this study focused on an older population with high SBP and low DBP and did not include subjects with DBP $\geqslant 90 \mathrm{~mm} \mathrm{Hg}$, it had a great advantage in that it could examine the effect of SBP and PP on the risk of decline of kidney function. However, it was unable to examine the effects of DBP and MAP on the risk of decline of kidney function. Schaeffner et al. ${ }^{23}$ also reported in the Physicians' Health Study that SBP and PP were stronger predictors of the risk of eGFR falling to $<60 \mathrm{ml} \mathrm{min}^{-1}$ per $1.73 \mathrm{~m}^{2}$. Their results were based on self-reported information, and direct measurements of BP, serum creatinine and proteinuria at baseline were not performed. Although the authors commented that self-reported SBP and DBP were highly correlated with measured SBP $(r=0.72)$ and DBP $(r=0.60)$, the correlation between PP and MAP calculated by self-reported SBP and DBP and PP and MAP calculated using the measured SBP and DBP must be lower than that between self-reported SBP and DBP and measured SBP and DBP. It was therefore difficult to compare the four BP components in the context of the risk of low eGFR using self-reported BP.

In the present study, we did not identify why DBP or MAP increased the risk of CKD. Two large-scale, collaborative cohort studies and other recent cohort studies have reported that DBP has a strong or stronger relationship to the incidence of coronary heart disease than SBP until age 50; $8,11,12,32,33$ thereafter, SBP and PP, but not DBP, were more influential. ${ }^{8,12,33-35}$ MAP was strongly related to the risk of coronary heart disease over a wide age range. ${ }^{8,12}$ For stroke, most prospective studies have reported that SBP is the most powerful risk factor, although these studies did not examine the effect of BP components on age-specific stroke incidence. ${ }^{15-17,35-38}$ Recently, three large-scale collaborative cohort studies and several large-scale cohort studies have examined BP components in relation to the risk of stroke by age group. ${ }^{8,11-14}$ These studies have reported that the relation of DBP to stroke incidence was as strong as or stronger than that of SBP until age $60-65 ; 8,11-14,39$ thereafter, the relation of SBP to stroke incidence was stronger than that of DBP. ${ }^{8,12-14,35}$ In addition, MAP was strongly related to stroke risk over a wide age range. ${ }^{8,11-14}$ However, PP was not associated with the risk of stroke after adjusting for MAP in both middle-aged and older subjects. ${ }^{13,14}$ These results suggest that each BP component might have a different role in organ damage at different ages. As our cohort was composed of subjects 40-55 years of age, our findings that both DBP and MAP were associated with an increased risk for kidney dysfunction were consistent with the results of previous reports of cardiovascular diseases in the middle-aged population. ${ }^{8,11-14,33,39}$ Further investigation is warranted to elucidate the age-specific hemodynamic mechanism of the initiation of CKD.

Our study has several limitations. First, the results might be biased because GFR was not directly measured. However, because direct measurement of GFR is cumbersome and expensive, this limitation is common in large cohort studies. Using the modification of diet in renal disease formula to estimate renal function should be permitted. ${ }^{40}$ Second, our study was based on the measurement of office 
BP at a single visit, not self-monitored BP, which may lead to a misclassification of BP levels. BP was measured once if the initial reading did not show hypertension, but was measured twice if hypertension was detected; this may also have led to a misclassification of BP levels. Third, because all participants were registered employees of the same company and from a single ethnic group, our results may not be representative of the general population. Instead, it may apply to middle-aged Japanese-American men and possibly other AsianAmerican and native Asian men. The results of the present study might underestimate the real associations between BP components and CKD because of the healthy worker effect. ${ }^{41}$ Fourth, the inclusion of some participants who started to take antihypertensive medication during the follow-up period may have contributed to an underestimate of the relationship between BP components and CKD risk. Fifth, calculated values of PP and MAP were used in place of the invasive measurement because it is not practical in a large-scale cohort study to use invasive measurement techniques. Any error that occurred as a result of these indirect measures, however, is likely to be random, as opposed to systematic, thereby biasing study results toward null values. ${ }^{42}$ Therefore, this might lead to an attenuation of the observed association between the BP components and the outcome.

These results provide evidence that both DBP and MAP are most predictive of the incidence of CKD in middle-aged Japanese men. Our findings supplement the many reports of the effects of BP components on cardiovascular disease and suggest that each BP component may have a different role in organ damage at different ages. Future clinical research is needed to examine whether DBP- and/or MAP-lowering therapy is effective in preventing or delaying the incidence of CKD.

\section{CONFLICT OF INTEREST}

The authors declare no conflict of interest.

\section{ACKNOWLEDGEMENTS}

This work was supported by a Grant-in-Aid for Scientific Research (17390177, 20390187) from the Ministry of Education, Culture, Sports, Science and Technology of Japan, as well as by facilities and services provided by the Kansai Health Administration Center at Nippon Telegraph and Telephone West Corporation. The funding sources had no role in the collection, analysis, or interpretation of the data or in the decision to submit the manuscript for publication. We thank the participants in the Kansai Healthcare Study for their dedication. This work was in part supported by a Grant-in-Aid for Scientific Research $(17390177,20390187)$ from the Ministry of Education, Culture, Sports, Science and Technology.

1 Chobanian AV, Bakris GL, Black HR, Cushman WC, Green LA, Izzo JL, Jones DW, Materson BJ, Oparil S, Wright JT, Roccella EJ, National Heart, Lung, and Blood Institute Joint National Committee on Prevention, Detection, Evaluation, and Treatment of High Blood Pressure; National High Blood Pressure Education Program Coordinating Committee. The seventh report of the joint national committee on prevention, detection, evaluation, and treatment of high blood pressure: the JNC 7 report. JAMA 2003; 289: 2560-2572.

2 Guidelines Subcommittee: 1999 World Health Organization-International Society of Hypertension Guidelines for the Management of Hypertension. J Hypertens 1999; 17: 151-183.

3 Tozawa M, Iseki $\mathrm{K}$, Iseki C, Oshiro S, Ikemiya $\mathrm{Y}$, Takishita S. Influence of smoking and obesity on the development of proteinuria. Kidney Int 2002; 62 956-962.

4 Jee SH, Boulware LE, Guallar E, Suh I, Appel LJ, Miller ER. Direct, progressive association of cardiovascular risk factors with incident proteinuria: results from the Korea Medical Insurance Corporation (KMIC) Study. Arch Intern Med 2005; 165: 2299-2304.

5 Yamagata K, Ishida K, Sairenchi T, Takahashi H, Ohba S, Shiigai T, Narita M, Koyama A. Risk factors for chronic kidney disease in a community-based population: a 10-year follow-up study. Kidney Int 2007; 71: 159-166.
6 Fox CS, Larson MG, Leip EP, Culleton B, Wilson PWF, Levy D. Predictors of new-onset kidney disease in a community-based population. JAMA 2004; 291: 844-850.

7 Obermayr RP, Temml C, Knechtelsdorfer M, Gutjahr G, Kletzmayr J, Heiss S, Ponholzer A, Madersbacher S, Oberbauer R, Klauser-Braun R. Predictors of new-onset decline in kidney function in a general middle-European population. Nephrol Dial Transplant 2008; 23: 1265-1273.

8 Lewington S, Clarke R, Qizilbash N, Peto R, Collins R, Prospective Studies Collaboration. Age-specific relevance of usual blood pressure to vascular mortality: a meta-analysis of individual data for one million adults in 61 prospective studies. Lancet 2002; 360: 1903-1913.

9 Miura K, Dyer AR, Greenland P, Daviglus ML, Hill M, Liu K, Garside DB, Stamler J. Pulse pressure compared with other blood pressure indexes in the prediction of 25-year cardiovascular and all-cause mortality rates: the Chicago Heart Association Detection Project in Industry Study. Hypertension 2001; 38: 232-237.

10 Inoue R, Ohkubo T, Kikuya M, Metoki H, Asayama K, Obara T, Hoshi H, Hashimoto J, Totsune $\mathrm{K}$, Satoh $\mathrm{H}$, Kondo $\mathrm{Y}$, Imai $\mathrm{Y}$. Predicting stroke using 4 ambulatory blood pressure monitoring-derived blood pressure indices: the Ohasama Study. Hypertension 2006; 48: 877-882.

11 Mosley WJ, Greenland P, Garside DB, Lloyd-Jones DM. Predictive utility of pulse pressure and other blood pressure measures for cardiovascular outcomes. Hypertension 2007; 49: 1256-1264.

12 Lawes CM, Bennett DA, Parag V, Woodward M, Whitlock G, Lam TH, Suh I, Rodgers A, Asia Pacific Cohort Studies Collaboration. Blood pressure indices and cardiovascular disease in the Asia Pacific region: a pooled analysis. Hypertension 2003; 42: 69-75.

13 Miura K, Soyama Y, Morikawa Y, Nishijo M, Nakanishi Y, Naruse Y, Yoshita K, Kagamimori S, Nakagawa H. Comparison of four blood pressure indexes for the prediction of 10-year stroke risk in middle-aged and older Asians. Hypertension 2004; 44: 715-720.

14 Miura K, Nakagawa H, Ohashi Y, Harada A, Taguri M, Kushiro T, Takahashi A, Nishinaga M, Soejima H, Ueshima H, Japan Arteriosclerosis Longitudinal Study (JALS) Group. Four blood pressure indexes and the risk of stroke and myocardial infarction in Japanese men and women: a meta-analysis of 16 cohort studies. Circulation 2009; 119: 1892-1898.

15 Brown DW, Giles WH, Greenlund KJ. Blood pressure parameters and risk of fatal stroke, NHANES II mortality study. Am J Hypertens 2007; 20: 338-341.

16 Bowman TS, Gaziano JM, Kase CS, Sesso HD, Kurth T. Blood pressure measures and risk of total, ischemic, and hemorrhagic stroke in men. Neurology 2006; 67: 820-823.

17 Inoue R, Ohkubo T, Kikuya M, Metoki H, Asayama K, Kanno A, Obara T, Hirose T, Hara A, Hoshi H, Totsune K, Satoh H, Kondo Y, Imai Y. Stroke risk of blood pressure indices determined by home blood pressure measurement: the Ohasama study. Stroke 2009; 40: 2859-2861.

18 Berne RM, Levy MN. Cardiovascular Physiology, 8th edn. Mosby-Year Book: St Louis, 2001, pp 135-151.

19 Safar ME. Pulse pressure in essential hypertension: clinical and therapeutical implications. J Hyperten 1989; 7: 769-776.

20 Benetos A, Laurent S, Asmar RG, Lacolley P. Large artery stiffness in hypertension. J Hyperten 1997; 15: S89-S97.

21 Franklin SS, Gustin W, Wong ND, Larson MG, Weber MA, Kannel WB, Levy D. Hemodynamic patterns of age-related changes in blood pressure: the Framingham Heart Study. Circulation 1997; 96: 308-315.

22 Young JH, Klag MJ, Muntner P, Whyte JL, Pahor M, Coresh J. Blood pressure and decline in kidney function: findings from the systolic hypertension in the elderly program (SHEP). J Am Soc Nephrol 2002; 13: 2776-2782.

23 Schaeffner ES, Kurth T, Bowman TS, Gelber RP, Gaziano JM. Blood pressure measures and risk of chronic kidney disease in men. Nephrol Dial Transplant 2008; 23: 1246-1251.

24 Sato KK, Hayashi T, Kambe H, Nakamura Y, Harita N, Endo G, Yoneda T. Walking to work is an independent predictor of incidence men of type 2 diabetes in Japanese: the Kansai Healthcare Study. Diabetes Care 2007; 30: 2296-2298.

25 Sato KK, Hayashi T, Nakamura Y, Harita N, Yoneda T, Endo G, Kambe H. Liver enzymes compared with alcohol consumption in predicting the risk of type 2 diabetes: the Kansai Healthcare Study. Diabetes Care 2008; 31: 1230-1236.

26 Harita N, Hayashi T, Sato KK, Nakamura Y, Yoneda T, Endo G, Kambe H. Lower serum creatinine is a new risk factor of type 2 diabetes: the Kansai Healthcare Study. Diabetes Care 2009; 32: 424-426.

27 American Diabetes Association. Diagnosis and classification of diabetes mellitus. Diabetes Care 2009; 32: S62-S67.

28 Matsuo S, Imai E, Horio M, Yasuda Y, Tomita K, Nitta K, Yamagata K, Tomino Y, Yokoyama H, Hishida A, Collaborators Developing J. Revised equations for estimated GFR from serum creatinine in Japan. Am J Kidney Dis 2009; 53: 982-992.

29 Katz MH. Multivariable analysis: a practical guide to clinicians. 2nd edn. Cambridge University Press: New York, 2006, pp 43-46.

30 Chatterjee S, Hadi AS. Regression analysis by example. 4th edn. John Wiley \& Sons, Inc: New Jersey, 2006 pp 287-327.

31 Armitage P, Berry G, Matthews JNS. Statistical Methods in Medical Research, 4th edn. Blackwell Publications: Oxford, 2002, pp 358-360.

32 Tverdal A. Systolic and diastolic blood pressures as predictors of coronary heart disease in middle aged Norwegian men. Br Med J (Clin Res Ed) 1987; 294: 671-673.

33 Franklin SS, Larson MG, Khan SA, Wong ND, Leip EP, Kannel WB, Levy D. Does the relation of blood pressure to coronary heart disease risk change with aging?: the Framingham Heart Study. Circulation 2001; 103: 1245-1249. 
34 Franklin SS, Khan SA, Wong ND, Larson MG, Levy D. Is pulse pressure useful in predicting risk for coronary heart disease? The Framingham Heart Study. Circulation 1999; 100: 354-360.

35 Psaty BM, Furberg CD, Kuller LH, Cushman M, Savage PJ, Levine D, O'Leary DH, Bryan RN, Anderson M, Lumley T. Association between blood pressure level and the risk of myocardial infarction, stroke, and total mortality: the cardiovascular health study. Arch Intern Med 2001; 161: 1183-1192.

36 Stamler J, Stamler R, Neaton JD. Blood pressure, systolic and diastolic, and cardiovascular risks: US population data. Arch Intern Med 1993; 153: 598-615.

37 Lindenstrøm E, Boysen G, Nyboe J. Influence of systolic and diastolic blood pressure on stroke risk: a prospective observational study. Am J Epidemiol 1995; 142: 1279-1290.
38 Nielsen WB, Lindenstrøm E, Vestbo J, Jensen GB. Is diastolic hypertension an independent risk factor for stroke in the presence of normal systolic blood pressure in the middle-aged and elderly? Am J Hypertens 1997; 10: 634-639.

39 Håheim LL, Holme I, Hjermann I, Leren P. Risk of fatal stroke according to blood pressure level: an 18-year follow-up of the Oslo Study. J Hypertens 1995; 13: 909-913.

40 Vassalotti JA, Stevens LA, Levey AS. Testing for chronic kidney disease: a position statement from the national kidney foundation. Am J Kidney Dis 2007; 50: 169-180.

41 Pearce N, Checkoway H, Kriebel D. Bias in occupational epidemiology studies. Occup Environ Med 2007; 64: 562-568.

42 Rothman KJ, Greenland S. Modern Epidemiology. 2nd edn. Lippincott Williams \& Wilkins: Philadelphia, 1998, pp 115-134. 